\title{
A Privacy Preserved and Credible Network Protocol
}

\author{
Zhongjiang Yao ${ }^{\mathrm{a}, \mathrm{b}}$, Jingguo $\mathrm{Ge}^{\mathrm{a}, *}$, Yulei $\mathrm{Wu}^{\mathrm{c}, *}$, Linjie Jian ${ }^{\mathrm{a}, \mathrm{b}}$ \\ ${ }^{a}$ Institute of Information Engineering, Chinese Academy of Sciences, Beijing, 100093, \\ China \\ ${ }^{b}$ School of Cyber Security, University of Chinese Academy of Sciences, Beijing, 100049, \\ China \\ ${ }^{c}$ College of Engineering, Mathematics and Physical Sciences, University of Exeter, Exeter, \\ EX4 $4 Q F, U K$
}

\begin{abstract}
The identities of packet senders and receivers are treated as important privacy information in communication networks. Any packet can be attributed to its sender for evaluating its credibility. Existing studies mainly rely on third-party agents that contain the packet sender's identity to ensure the sender's privacy preservation and credibility. In this case, packet senders run the risk that their privacy might be leaked by the agent. To this end, this paper proposes a Privacy Preserved and Credible Network Protocol (PCNP), which authorizes the agent to hide the identities of senders and receivers, while guaranteeing the credibility of a packet. The feasibility of the PCNP deployment is analyzed, and its performance is evaluated through extensive experiments.
\end{abstract}

Keywords: Privacy Protection, Credibility, Accountability, Network Protocol

\section{Introduction}

The research on privacy protection and credibility has made significant progress in the areas such as Internet-of-Things(IoT) 1, 2, 3, 4, battery vehicles [5, 6, big data [2, 7, Blockchain [8, etc., but it has been relatively 5 slow for the whole Internet. The current Internet architecture lacks security considerations in its initial design. With the rapid development of computer and communication technologies, the Internet becomes more complex, and its security is severely challenged. The pervasive use of the Internet has caused an incredible growth of unwanted traffic, such as spam, malware and malicious 10 intrusions [9]. The Cybercrimes are constantly growing, and the global Cybersecurity market is expected to skyrocket to $\$ 231.94$ billion by 2022 [10. In terms of security, the packet owner (e.g., packet sender) always wants to show its credibility while keeping its privacy (i.e., the identity of packet sender) preserved

\footnotetext{
* Corresponding author

Email addresses: gejingguo@iie.ac.cn (Jingguo Ge), y.l.wu@exeter.ac.uk (Yulei Wu)
} 
[1]. For example, in a typical client-server $(\mathrm{C} / \mathrm{S})$ communication scenario, the 15 server endeavors to have its identity hidden to avoid being attacked, while providing a credible service; on the other hand, the client expects to make the server trust its service request, without showing its identity. However, it is a great challenge for a sender and/or a receiver to be credible while keeping its privacy preserved.

Privacy Preservation refers to the situation that no one else except the sender itself knows its identity [12, 13, 14, when a packet is issued out; no one else except the receiver itself knows its identity [15, 16, when a packet is received; and no one can learn the payload except for the sender and the receiver [15. All three types of network layer privacy data have been proposed in [17.

${ }_{25}$ The packet sender and receiver are threatened with privacy leak due to the risks such as malicious network monitoring or attack.

Credibility indicates the authenticity and accountability of packet senders [18. It is worth noting that it is different from the credibility of web page links 19. In other words, the packet sender holds the responsibility for ensuring the 30 accountability of the packet it provides. Anyone receiving the packet can verify its authenticity by challenging its source (i.e., packet sender) [20].

Existing researches mainly focus on either the privacy preservation or the credibility guarantee of packet senders, typically offering one by sacrificing the

35 other. Recent studies, e.g., Accountable and Private Internet Protocol (APIP) [21] and Accountable and Private Network Architecture (APNA) 22], are to find a balance by making a tradeoff between these two factors. However, they focused on using third-party agents to ensure the privacy preservation and the credibility guarantee for packet senders, where the sender's identity must be 40 acquired by the agent. Under this circumstance, the packet senders run the risk that their privacy might be leaked by the agent.

To overcome this important problem, this paper proposes a Privacy Preserved and Credible Network Protocol (PCNP). The main contributions of this paper are summarized as follows:

- A Communication and Verification Agent (CVA) is proposed to ensure the privacy preservation of packet senders and receivers. Instead of using the sender's and receiver's identities in packet's source and destination fields, the CVA's identities are used. The packet transmission between senders and receivers thus looks like being forwarded between their associated CVAs.

- Considering the risk of privacy leak by the CVA, a temporary anonymous identity, $A m I D$, is proposed. AmIDs are published to CVA for hiding sender's and receiver's identities, while CVA knows nothing about the owner of AmID. In other words, the sender can anonymously authorize 
the CVA to guarantee the credibility of a packet issued by it, without the risk of leaking its identity.

- New credibility metrics are proposed to measure the degree of the sender's credibility. The victim determines the blocking time of malicious traffic according to the credibility metrics.

- The deployment and security considerations of the proposed PCNP is discussed. Its performance is evaluated through extensive experiments. The results demonstrate the feasibility and merits of the proposed protocol.

The remainder of this paper is organized as follows. Section 2 outlines the adversary models for privacy preservation and credibility. Section 3 gives an overview of the proposed PCNP. The detailed design is discussed in Section 4. Section 5 analyzes its potential security issues. Section 6 presents the compatibility and deployment considerations of PCNP. Section 7 analyzes its performance. The related work is shown in Section 8. Finally, Section 9 makes a conclusion.

\section{The Motivation and Adversary Models}

\subsection{Motivation}

Frequent Internet security incidents have resulted in serious problems for both the sender and the receiver of messages. Under this circumstance, the sender needs to pay more attention to its privacy protection and manage to 75 minimize the information leakage (such as IP address, communication relationship, etc.) in the communication process; in addition, it promotes the recipient's need for receiving secure and reliable messages. Malicious traffic pursues the sender's responsibility, especially for the traffic that causes serious damage.

It is known from [23] and the TCP/IP network protocol stack that different

so privacy information needs to be protected at different levels. Privacy protection during network communication is usually to hide the user's node identity/location information. These information are used for packet routing, so the protection of these information belongs to the network level. Privacy protection at the application layer can only protect such as application type, data informa-

85 tion and user activity patterns 24, and the application layer cannot verify the connection between the application layer part and the node identity/location. The node identity/location information is hidden at the data link layer, i.e., a MAC address, and there is little to do with the node identity/location information in the cross-network routing message.

$90 \quad$ In order to hide node identity/location information, various anonymous communication networks have been proposed at the network level, but they only have privacy protection capabilities and no accountability functions; technologies with accountability in network layer are rarely developed. The sender wants his/her privacy to be protected, while the receiver wants to receive packets that

95 are authentic. In order to protect both senders and receivers, both privacy 
protection and accountability are indispensable. With the rapid development of network security techinques, how to balance privacy protection and accountability has become the most urgent issue.

\subsection{Adversary Models}

\subsection{The Basic Design of PCNP}

PCNP is designed to provide the credibility of packets, while keeping the privacy preserved for packet senders and receivers. To describe the principle of the proposed PCNP, the key symbols used are shown in Table 1. In the 
proposed PCNP, a temporary identity called $A m I D$ is introduced for anonymous communication between the CVAs of the sender and the receiver. The AmID is used to reduce the risk of leaking sender's identity by the CVA, which will be detailed in Section 4. In order to ensure the packet to be delegated is credible, we need to redesign the packet formation, which is described in Section 3.1.2.

Table 1: The Symbols Used in The PCNP Description.

\begin{tabular}{l|l}
\hline Symbols & Description \\
\hline$S K_{S}, S K_{D}, S K_{V}$ & $\begin{array}{l}\text { Symmetric keys shared by CVA and PKM, CVA and } \\
\text { delegated host, CVA and routers, respectively }\end{array}$ \\
$E_{S K}$ & $\begin{array}{l}\text { Encrypted with symmetric key SK } \\
E_{P K M}, E_{C}, E_{S}\end{array}$ \\
seed & Encrypted with public key of PKM/client/server \\
SerID & Tsed to search symmetric key or identity of sender \\
& The service identity in packet header, like port num- \\
$F p(D)$ & The fingerprint of data $D$ (i.e., packet, ID) \\
$H(D)$ & The hash of data $D$ \\
$C e r t i f i c a t e$ & The certification that server authorizes CVA to reg- \\
& ister service to name resolution server \\
$M$ & Encrypted Certificate with the public key of PKM \\
$C$ & The collection of AmID sent to CVA by receiver \\
$S I G(P)$ & The signature of packet $P$ signed by its source \\
$N a m e$ & The name of a service \\
$E x p$ & The symbol of deadline \\
\hline
\end{tabular}

\subsubsection{Packet Address Format}

The address format proposed in PCNP consists of two logical pieces: a Network IDentity $(N I D)$ identifies a network, which is used to forward packets to the destination network, and a Host IDentity (HID) identifies a host in the des145 tination network. The complete address format can be expressed as NID:HID. Each host contains two types of HID: one is Real IDentity $(R I D)$, which is generated by using the public key that cannot be tampered and repudiated; the other is Anonymous IDentity $(A m I D)$ which is generated by using $R I D$, MAC, random number and timestamp. $A m I D$ is used between CVA and packet sender or receiver (more details will be given in Section 4). RID is long-term effective, while $A m I D$ is temporary which is used only once.

NID:RID is a traditional address in a sense, identifying a host, which is globally unique and routable. However, NID:AmID is a temporary identity, but in the same format of NID:RID. To prevent the conflicts with the AmID 155 generated by the other hosts in the same network domain, $A m I D$ should be registered with the Public Key Manager (PKM) for validity check. This check has to guarantee: 1) $A m I D$ is generated by the packet sender; 2) $A m I D$ is 


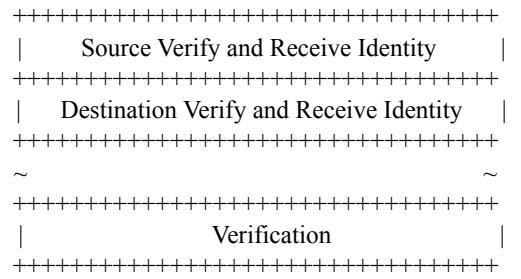

Figure 1: The Packet Format in PCNP.

not being occupied. As a temporary identity, AmID has a life-time from the registration to the end of its usage. If its life-time is over, or it has been used, $A m I D$ will be removed from the AmID Cache (AC).

As a host may contact multiple hosts for a certain period of time or maintain multiple sessions with another host, it needs to generate multiple $A m I D$ s for privacy preservation. A network-layer protocol needs to implement an AC to save all AmIDs currently registered. When receiving an Address Resolution Protocol (ARP) packet, the host checks if the HID is RID first. If it is, the host responds with its MAC address. Otherwise, the host searches the AC. If the AC contains the HID, the host will return its MAC address; otherwise it ignores the ARP.

\subsubsection{Packet Format}

To hide the identities of sender and receiver, PCNP replaces the source address, in traditional packet header, with the Verify and Receive Identity (VRI) of source and replaces the destination address with destination VRI, as shown in Figure 1. The source VRI is used to verify the authenticity of packets, and the destination VRI is used to forward the packet to its receiver. Rather 175 than identifying the sender and the receiver, the source VRI identifies the CVA who vouches for the packets, and the destination VRI identifies the CVA who delegates the received packets. If the communicators are not anonymous, the VRIs are filled with the NID:RID of the sender and the receiver. A Verification field (Ver) is introduced for two roles: one is used to verify the authenticity of packets, and the other is used to challenge the packet sender [25]. The Ver consists of $\operatorname{seed}_{S}$ and the signed fingerprint of payload $S I G_{S}(F p(P))$. The $\operatorname{seed}_{S}$ is encrypted with $\operatorname{Exp}_{S}$ by the public key of PKM which is shared by sender and PKM. The seed $_{S}$ is used to ensure the validity of the packet, and the $S I G_{S}(F p(P))$ is mainly used to authenticate the identity of the sender of the packet.

$$
\left[E_{P K M}\left(\operatorname{seed}_{S}, \operatorname{Exp}_{S}\right), S I G_{S}(F p(P))\right]
$$

$\operatorname{see}_{S}$, which is refreshed periodically, is a secret token generated by the sender. If a perpetrator generates a public key conflicting with that of an existing host, the packets sent by the perpetrator will be failed for verification, as the perpetrator knows nothing about the $\operatorname{see}_{S}$. 




Figure 2: An Overview of PCNP.

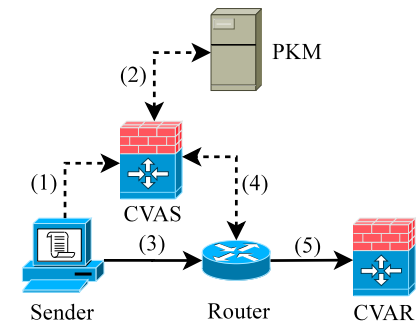

Figure 3: The sending process: (1) Sender sends Brief to CVAS; (2) CVAS verifies the authenticity of the Brief; (3) Sender sends packet; (4) Router verifies the authenticity of the packet; (5) Router forwards the packet.

\subsubsection{Communication}

The overview of PCNP is shown in Figure 2. The Sender's CVA (CVAS) and the Receiver's CVA (CVAR) are chosen by the sender and the receiver, respectively, which are used to hide their identities. PKM is the facility used to manage public keys and provide authentication, which prevents host from generating public keys randomly. To describe the PCNP clearly, the complete communication process is divided into three parts: sending process, receiving process, and accountability process.

\section{(1) Sending process.}

As shown in Figure 3, to keep the credibility of a packet while hiding the sender's identity, CVAS is introduced to establish the relationship between packet and its sender. Before sending a packet $P$, the sender will anonymously send a Brief, which is a packet carrying the summary of $P$, to the CVAS with $A m I D$. For the authenticity of Brief, CVAS needs to verify its authenticity from PKM. At the same time, the sender sends out $P$. Due to the lack of sender's identity, the routers on the forwarding path will challenge the credibility of $P$. To do this, routers can send Verify, which is the packet used for verifying the authenticity of $P$, to the CVAS which caches the summary of $P$ (more details will be provided in Section 4). If every packet of a stream gets verified, the forwarding performance will be seriously affected (i.e., the speed of packet transition will be slowed down, and network congestion may be produced). Therefore, a 


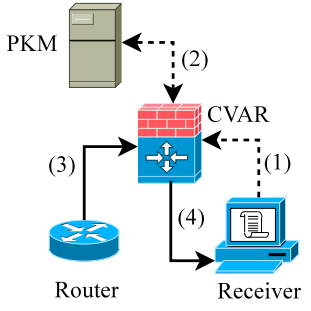

Figure 4: The receiving process: (1) Receiver sends a Publish to CVAR; (2) CVAR verifies the authenticity of Publish; (3) Router forwards a packet to CVAR; (4) CVAR forwards the packet to receiver after being processed.

local whitelist is introduced to cache the summary of packet that has passed the verification.

\section{(2) Receiving process.}

A receiver can be a server which provides certain services (e.g., website and cloud computing) or a host which receives a response. As shown in Figure 4, if it is a server, it will anonymously send Publish to CVAR with AmID to hide its identity (more details are shown in Section 4). A Publish is a packet used to authorize its CVAR to register a service to Domain Name System (DNS) with the NID:RID of CVAR. Due to the lack of the receiver's identity in the Publish, 220 CVAR needs to verify the authenticity of Publish. When requesting a service of receiver, the sender issues a request $P$ to CVAR. CVAR forwards $P$ to the receiver with $A m I D$. If it is the host to receive a response, CVAR receives the response packet and forwards it to the receiver with $A m I D$.

\section{(3) Accountability process.}

${ }_{225}$ As shown in Figure 5, when receiving unwanted traffic (e.g., malicious traffic), the receiver sends Shutoff to decrease the resulting negative impact. The Shutoff is the packet used to report to CVAS and block unwanted traffic quickly. During the forwarding of Shutoff, routers can verify it as a standard packet as mentioned in the Sending process. When CVAS receives a Shutoff, it finds the related records and stops providing the verification of related packets, and finally forwards the encrypted Shutoff to the sender. The sender should then stop sending the traffic. A malicious host may ignore the Shutoff and the negative impact can continue spreading. But the CVAS can fail the packet verification and stops the traffic. To achieve better performance, the router introduces a 235 Blacklist, which caches the blocked packets.

The proposed PCNP has four advantages. First, CVA protects the privacy of senders, although CVAS is not clear to whom it delegates. Second, it protects the privacy of receivers, although CVAR is not clear of those delegated receivers. Third, PCNP hides the communication relationship between sender 240 and receiver. Finally, it provides a credible communication while protecting the privacy of senders and receivers. 




Figure 5: The accountability process: (1) Receiver sends the Brief of Shutoff; (2) Receiver sends Shutoff; (3) Router verifies the Shutoff; (4) Router forwards the Shutoff to CVAS; (5) CVAS forwards the processed Shutoff to sender.

\section{The Details of PCNP}

This section shows the detailed design of PCNP, where the design of anonymity method, key management and other mechanisms are inspired by [26] in Chapter

245 15. It should be noted that all packets in PCNP contain Ver. For the sake of the clarity of illustration, the Ver is not included in describing a packet in this section.

\subsection{Communication and Verification Agent (CVA)}

CVA plays a key role in the proposed PCNP. It proves the relationship between packet and its sender/its receiver.

\subsubsection{The relationship between packet and its sender}

To prove the relationship between the packet $P$ and its sender, the sender issues a Brief with NID:AmID to CVAS before sending $P$, which can be used as digital certificate if legal intervention is required. The Brief contains the 5-tuple (i.e., source VRI, source SerID, protocol type, destination SerID, and destination VRI) and the fingerprint of $P$. SerID is selected from the SerID collection, which is negotiated between CVA and the delegated host. To prevent the anonymity scope of senders from being narrowed by others using the link between 5-tuple and NID, we suggest that the payload of Brief should be 260 encrypted using symmetric key $S K_{D} . S K_{D}$ is negotiated after the sender establishes a connection with CVAS using $A m I D$, as shown in Figure 6. When receiving a Brief, CVAS needs to verify its authenticity from PKM (more details are given in Section 4.2.1).

The sender sending Brief to CVAS can be expressed as:

$$
\operatorname{Brief}\left(E_{S K_{D}}\left(5-\text { tuple, } S I G_{S}(F p(P)), A m I D\right)\right)
$$

\subsubsection{Forward packets to the receiver}

The basic function of CVAR is to forward packets to receivers. As mentioned in Section 3, receivers can be divided into two types: a server (i.e., web sites, cloud computing), from which a sender requests a service; and a host, who receives responses. If the receiver is a server, it authorizes CVAR to register its 
(1) Publish service.

Publish The receiver sends Publish to authorize CVAR to register services with the NID:RID of CVAR. Publish is a packet for authorizing, which contains the service name, encrypted authorization Certificate, and a collection of AmIDs. The Certificate is the credential that a server authorizes CVAR, which has been uploaded and confirmed by PKM. The Certificate contains the service name, the identity of server, and the identity of CVAR to be authorized. For the credibility of Publish, the service name, encrypted Certificate, and the hash of collection of $A m I D$ s will be sent to PKM for verification (more details

280 will be available in Section 4.2). The AmID collection is used for CVAR to forward requests with NID to the server as the destination VRI. Each service has an $A m I D$ collection, and each $A m I D$ is assigned to a request. The payload of Publish is encrypted with $S_{D}$ which is shared between CVAR and server.

The receiver authorizing CVAR to register service can be shown as:

$$
\operatorname{Publish}\left(E_{S K_{D}}(\text { Name }, M, C)\right)
$$

Update The size of AmID collection is limited and needs to be updated periodically. This paper proposes two options for updating: Interval-based update and Quantity-based update.

Interval-based Update. Considering that some servers may have less traffic, AmID collection may have not been used up in updating interval, interval. As

290 the $A m I D$ runs the risk of being leaked, and the sender loses its anonymity beyond the update interval, the AmID collection is still updated periodically. Therefore, each server has a Timer. When there is time $T$ left before the interval ends (interval < life-time), the server starts to generate a new AmID and updates them to CVAR when time $\tau(\tau<T)$ is left. The update is anonymous, so it needs to be verified similar to the Publish.

Quantity-based Update. Considering that some servers may have amounts of traffic, and the AmIDs in life-time may be used up, PCNP provides a quantitybased update mechanism. Each server sets a Counter to record the number of remaining $A m I D$ s. When Counter reaches the threshold $\theta(\theta \geq 1)$, the server 300 updates $A m I D$ s to CVAR. The update will be handled in the same way as the Interval-based scheme.

\section{(2) Forward packet.}

The destination VRI in the packet is a CVAR's NID:RID, which is not the final receiver. CVAR should forward packets to its final receiver.

To avoid observer's tracking, CVAR establishes a new connection with delegated receiver using a new $A m I D$. If the packet is a request to a server, $A m I D$ is selected from the $A m I D$ collection; if the packet is a response, $A m I D$ comes from a Brief record. Then, CVAR and the delegated receiver negotiate a symmetric key $S K_{D}$. The CVAR re-encapsulates the packet using $S K_{D}$. 


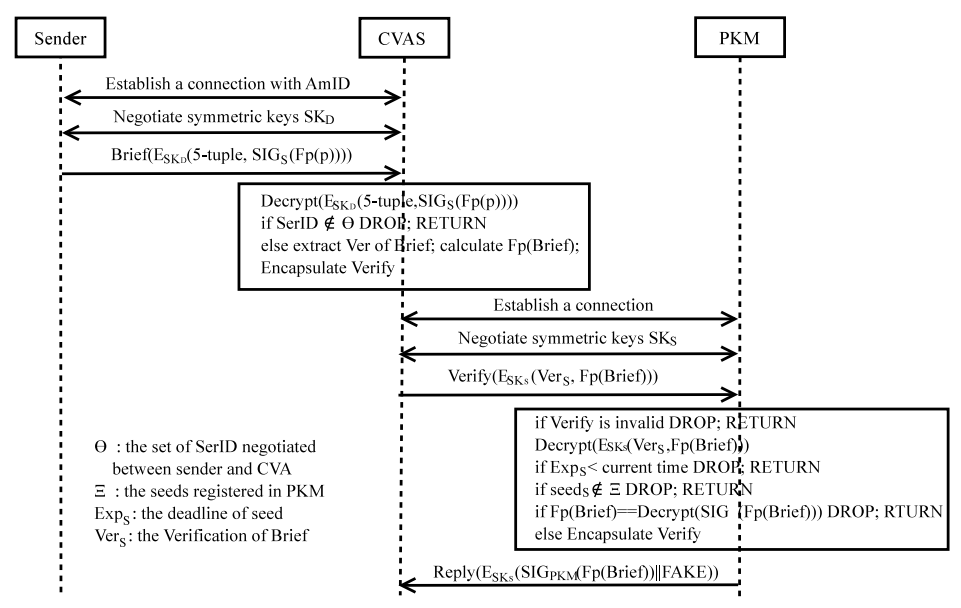

Figure 6: The process of Brief.

310 encrypts the Ver of Publish with $S K_{S}$ and encapsulates it into Verify. Then the CVAR sends the Verify to PKM for verification. The PKM mainly verifies four things: 1) the Verify is valid; 2) the Ver of Publish is valid; 3) the Certificate 


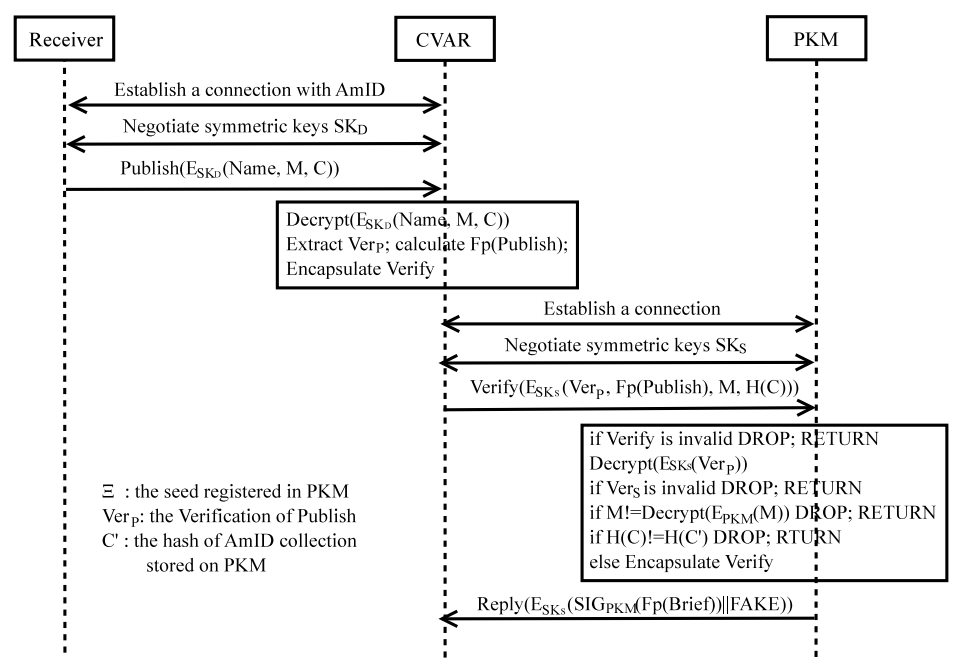

Figure 7: The verification of Publish.

335 matches with a registered one in PKM; and 4) the $H(C)$ matches with the hash of the registered AmID collection. If all the conditions are satisfied, PKM returns the fingerprint of Publish signed by PKM, and CVAR registers the service to DNS. The details are shown in Figure 7.

(3) Routers verify authenticity of packet to be forwarded.

When receiving a packet, routers check their Whitelist in which the packets have been verified. If it is not in, routers search the Blacklist in which the packet is blocked. If it is blocked, routers drop the packet directly. When the packet is neither in Whitelist nor in Blacklist, routers send Verify to verify the packet authenticity (see Figure.8). If the packet is credible, routers continue 345 forwarding it.

\subsubsection{Accountability}

A receiver should only account for unwanted packets for any damages.

\section{(1) Shutoff.}

Shutoff is used to block malicious traffic of the sender. However, the ma-

350 licious sender may ignore the Shutoff. Therefore, we use routers and CVA to play a supporting role. Once receiving a Shutoff, routers search the information carried in the Shutoff in the local Whitelist. If it is not in, routers put it in their Blacklist; if it is in, routers confirm the Shutoff from the CVAR. If the Shutoff is valid, it will be removed from Whitelist and added into Blacklist; otherwise 355 routers ignore the Shutoff. Once being found in Blacklist, the packet will be discarded. 


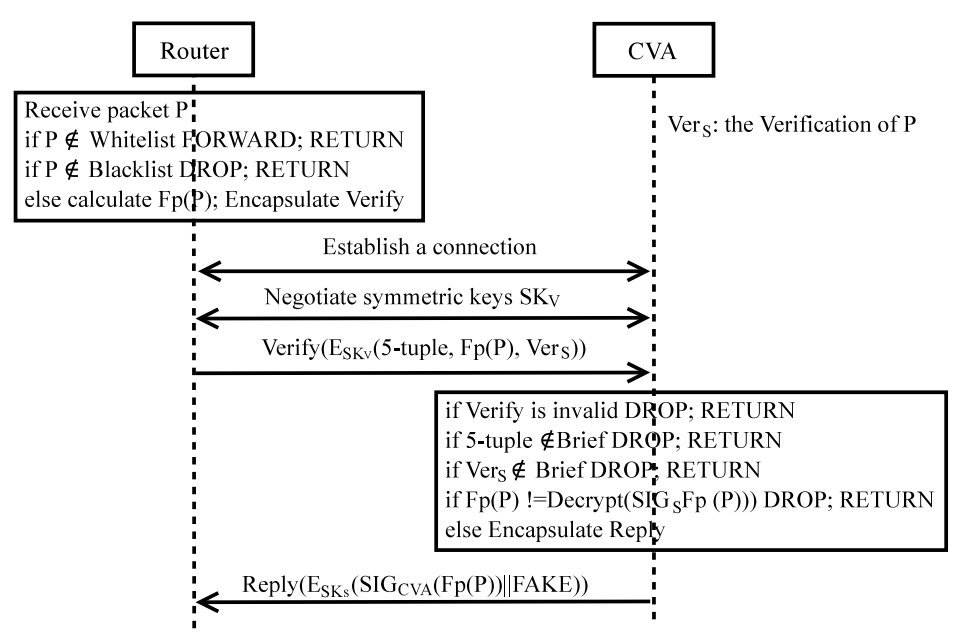

Figure 8: The verification of a packet.

Granularity The traditional way uses the 5-tuple in packet header to form Flow Identity. The SerID is one component of 5-tuple in PCNP like port number. When several senders use the same proxy, it is difficult to distinguish those access to the same service on the same server for the 5-tuple, if they use the same source SerID, destination SerID and VRI. To provide finer-granularity traffic accountability, CVA and sender (or receiver) negotiate SerID collection with $A m I D$. Different traffic will have different SerIDs: a sender can use a SerID to a host, and it can also use a SerID to a session, even to a packet.

Credibility metrics In APIP, long-term fix and short-term fix are proposed [21, which lacks flexibility. Therefore, we propose new credibility metrics: the Damage Degree (DD) $\psi$, the Historical Safety Assessment (HSA) $\Phi$, and Benign Ratio (BR). DD is calculated according to resource consumption, performance influence, and data damage. HSA obeys a power function with a base of mean value of historical $\Psi$, multiplied by a constant coefficient to make the value range between $[0,1]$, which represents the reputation of a node. BR is the ratio of the benign packets $\delta$ and total volume of packets $\Sigma$. DD, HSA and BR are typical metrics that can be used to evaluate sender reputation, and they are the basis for determining the blocking time. The higher the DD and the lower 375 the HSA, the longer the blocking time by Shutoff is. When receiving unwanted traffic, the receiver determines the blocking time $\Gamma$ for the sender to fix the problems, according to the credibility metrics which are computed in PKM as follows.

$$
\Gamma=\left(\psi+\left(\frac{\delta}{\Sigma} \cdot \Phi\right)^{-1}\right) \cdot \Omega, \mu<\frac{\delta}{\Sigma} \cdot \Phi<1,1 \leq \psi \leq 10
$$

where $\Omega$ is the time-unit of blocking time. We evaluate the sender's reputation by calculating $\frac{\delta}{\Sigma} \cdot \Phi$ and set a threshold $\mu$ as a criterion for evaluating sender 
reputation. When the reputation is lower than $\mu$, all traffic of the sender is permanently blocked; otherwise, the blocking time is calculated according to the above formula.

Security NIC Perpetrators usually do not admit their malicious actions

and even ignore Shutoff. We therefore introduce a Security Network Interface Card (S-NIC). S-NIC is assembled on the host using hot swapping and burned asymmetric key and identity. Host cannot modify them via Operating System (OS) and malicious programs. S-NIC's built-in cache space is used to cache fingerprint of packets sent through it. S-NIC checks the Ver of the packet sent out through this S-NIC. Once receiving a Shutoff, OS stops the application, and S-NIC filters related packets.

\section{Security Analysis}

\subsection{Bootstrap Trust}

Although PCNP uses public key to generate self-certifying identity $(R I D)$, additional supports are required for credibility. Brief contains the sender's signature to prove its authentication and encrypted with symmetric key for the privacy of senders. Except for those mentioned in Brief, Publish carries an encrypted credential for authorizing CVA to register service. Verify includes two categories to keep the credibility: first, CVA verifies the authenticity of delegated information; second, anyone can verify the authenticity of packets from the CVA. The payload of Verify is also encrypted with symmetric key. Shutoff contains Ver. If there are any doubts about a Shutoff, the sender can verify it from CVA, who caches the Brief of the Shutoff.

\subsection{Attack on Credibility}

405

\subsubsection{Spoofing}

Perpetrators have two possible spoofing packets. First, intercepting a packet and reinjecting it into network for replay attack. Records in Whitelist have a lifetime. If they are outdated when replay attack happens, the verification will fail; if they are within time limits, the receiver sends Shutoff. Second, perpetrators generate public key and identity randomly. If the public key generated has not been registered in PKM, the verification of the Brief signed by this public key will be failed, and the CVAS will not store its record. If the public key has registered in PKM, the Brief verification will be failure as the perpetrator knows nothing about the secret token.

\subsubsection{Risk of $C V A$}

If perpetrators send Brief with false HID, the verification will be failed as the seed cannot be known by perpetrators. Perpetrators can also send a large amount of false Brief or false packets causing routers to send a lot of Verify which may lead DoS attack and make CVA lose service capability. Although CVA has higher reputation, it still can be compromised. A compromised CVA 
may fail a valid packet or authenticate an invalid packet. In this case, it is worth noting that the CVA can be replaced freely when sender or receiver detects these malicious actions, because the correspondence between CVA and server is not fixed.

\subsection{Attack on Privacy}

The goal of privacy preservation is to protect the relationship between packet and its sender (or receiver), the communication relationship between sender and receiver. In APIP 21 and APNA 22, the privacy can be available as long as the agent is compromised. While in PCNP, all records in CVA, which is the agent containing only AmID, do not contain sender's or receiver's identity, which means the privacy mentioned above cannot be leaked. Though AmID can be obtained via CVA, it is not sure to whom AmID belongs.

\section{Deployment and Compatibility}

\subsection{Deployment}

When deploying PCNP, a highly trusted third-party needs to be selected as a CVA (e.g., ISP, which is short for Internet Service Provider, or a company with high reputation). In current network, Network Address Translation (NAT) and Border Router (BR) enjoy good reputations. When receiving a packet, CVA replaces the destination address with $A m I D$, which is similar to NAT. When a

440 packet leaves its source domain, NAT can provide verification and accountability for the packet through it. In the Autonomous System (AS), the BR, which is the AS gateway for packet transition, can record all packets through it and prove the authenticity of packets within it. That is to say, both NAT and BR can be used as CVAs.

\subsection{Compatibility}

If PCNP is introduced into the widely used TCP/IP network, the works below are needed: deploy CVAs; add additional functions to PKI; use source and destination addresses in packet header as source and destination VRI; and fill Ver in optional field of IP header. It is worth noting that the NAT or BR can be configured as the CVA. In order to ensure IP addresses are credible and comply with PCNP, PKI needs to add seed, authorization credential and verification function. In a Local Area Network (LAN), many IP addresses with the same NID must be reserved as NID:AmID. ISPs can dynamically allocate IP addresses as NID:AmID by DHCP. IPv6 is considered as the next generation Internet protocol and possesses a lot of similarity with IPv4. IPv6 can be tuned in the same way as IPv4 when PCNP is introduced. In addition, some wellknown projects on future Internet architecture, e.g., MobilityFirst [27] and HIP [28, have investigated the way of adapting to the changes of the future traffic types. MobilityFirst and HIP introduced the flat naming scheme. MobilityFirst separates host identity from location with Globally Unique Identifier (GUID). 
PCNP only concerns how to hide GUID without worrying about Network Address (NA). In order to use the proposed PCNP in MobilityFirst, we need to deploy CVAs, replace source and destination GUIDs in packet with the GUIDs of CVAS and CVAR, and add Verification in Service Header Extensions. As HIP packet header is IPv6 header, it can follow IPv6 adjustment method to use PCNP.

\section{Evaluation and Performance Analysis}

To evaluate the proposed PCNP, this paper uses IP as overlay. The sender, router and receiver are implemented with $\mathrm{C}++$ for faster packet forwarding; the 470 CVA and PKM are implemented with JAVA for multi-platform compatibility. The experiment is made on two physical computers: the OS is Ubuntu 16.04 whose core is 4.4.0-83-generic, and CPU is i5-3470T @ 2.9GHz *4. The sender, router and receiver run as processes on one computer, while CVA and PKM run as processes on the other one.

\subsection{Round-Trip Time (RTT)}

$\mathrm{RTT}$ is the time from sending a request by the sender to receiving its response. It directly affects how users feel the network they used subject to a given network protocol and is one of the most important indicators for evaluating the feasibility of a network protocol. In this experiment, we change two variables: the number of routers on the forwarding path and the number of routers verifying the authenticity of packet. Figure 9 depicts the RTT between sender and receiver, whose $x$-axis represents the number of routers and the $y$ axis denotes the RTT. For each additional router forwarding on the routing path without verification as what the Internet Protocol (IP) does, one link transmission time $T_{t}$ and one router processing time $T_{p}$ are required. The total time is $T_{\text {sum }}=\sum_{i=h}^{I}\left(N \cdot\left(T_{t}^{i}+T_{p}^{i}\right)+T_{p}^{i}\right)$, where $I$ is the total number of packets. From this figure, we can see that the RTT is increasing with the increase in the number of routers on the forwarding path or the number of routers providing verification. It takes longer time for RTT to go through routers with verification 490 than routers only forwarding packets. But the time used for verification does not obviously increase with the number of by-passing routers. The $i$ th packet verification time required for the router is $t^{i}$. The total time with verification is $T_{\text {sum }}=\sum_{i=h}^{I}\left(N \cdot\left(T_{t}^{i}+T_{p}^{i}+t^{i}\right)+T_{p}^{i}\right)$. However, due to the introduction of whitelist and the blacklist, only the first one packet needs to be authenticated by the routers of the path, and the subsequent packets only need to access the blacklist and whitelist of the routers. This time for accessing the blacklist and the whitelist is much shorter than the packet verification time, and then can be ignored. Thus, the total time with verification also can be expressed as $T_{\text {sum }}=\sum_{i=h}^{I}\left(N \cdot\left(T_{t}^{i}+T_{p}^{i}\right)+T_{p}^{i}\right)$. Therefore, the RTT is acceptable in reality as the value is millisecond. 


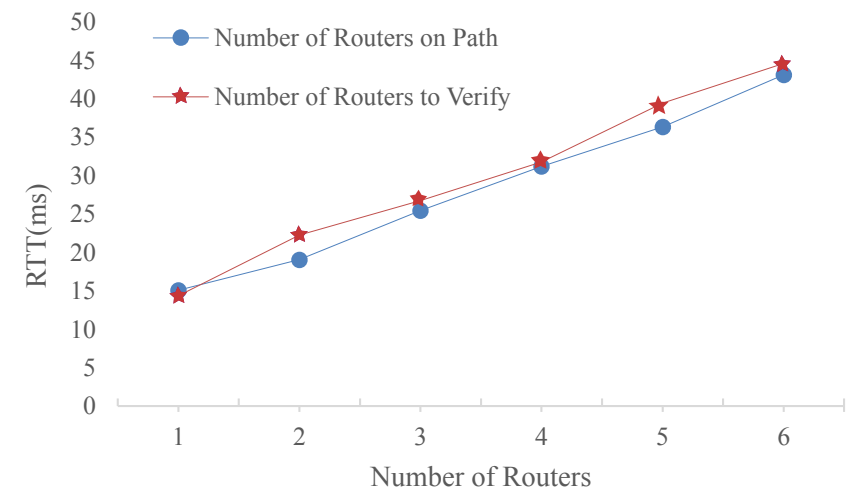

Figure 9: The RTT of a communication between the sender and the receiver

\subsection{AmID Collection}

AmID Collection is an important part of PCNP privacy protection. The frequency of $A m I D$ Collection update and the size of $A m I D$ Collection not only affect PCNP's ability to protect privacy but also affect network congestion.

505 The relationship among the size of AmID collection, lifecycle and traffic needs to be taken into consideration. We use RSA1024 and SHA256 to generate $H I D$. Assuming that the traffic volume changes between 10 and 1,000,000, and the lifecycle varies from 12 to 72 hours. Figure 10 depicts the space usage of CVAR, whose $x$-axis is the access traffic volume that represents the volume of received packets by the receiver, the $y$-axis denotes the lifecycle, and the $z$-axis represents the space usage. From Figure 10, we can see when traffic is constant, $A m I D$ collection size is linearly positive correlated to lifecycle. When lifecycle is constant, the size of $A m I D$ collection is linearly positive correlated to traffic.

When the access traffic volume is constant, the longer the AmID Collection update cycle is, the more $A m I D$ s needs to be included in the $A m I D$ Collection in order to ensure normal service delivery, but the longer update cycle also increases the risk of $A m I D$ being leaked. When the $A m I D$ Collection has a fixed capacity, the larger the volume of access is, the smaller the update cycle is ${ }_{520}$ required by the $A m I D$ Collection, but the risk of $A m I D$ being leaked is reduced. When the AmID Collection update period is fixed, the AmID Collection update cycle will be shortened in order to maintain normal service capabilities.

\subsection{Blocking Time}

Blocking unwanted traffic is a specific punishment for PCNP to account for malicious users, and is a direct indicator that affects the credibility of PCNP. DD is divided into 10 grades according to the degree of damage. BR changes between 0.9 and 1 , and $\mu=0.9$. HSA varies between 0 and 1 . The blocking time is the period the traffic to be blocked. The Growth Unit of DD, HSA and BR 


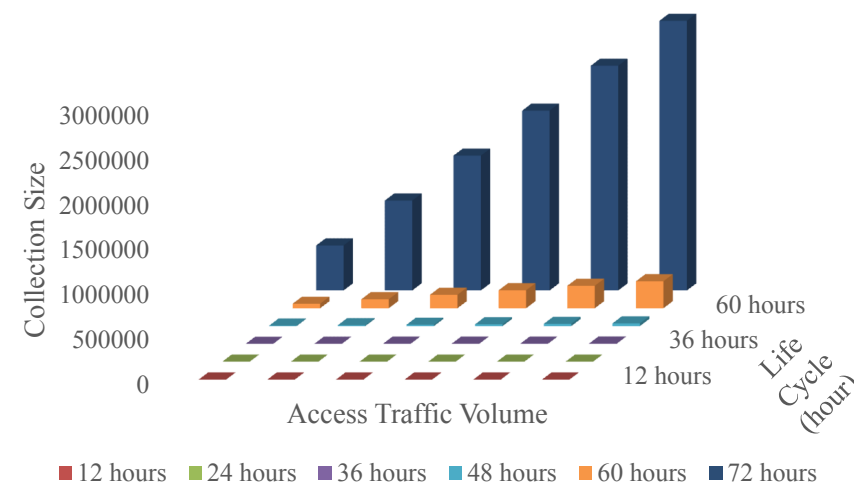

Figure 10: The relationship between $A m I D$ Collection and AmID's lifecycle, access traffic volume

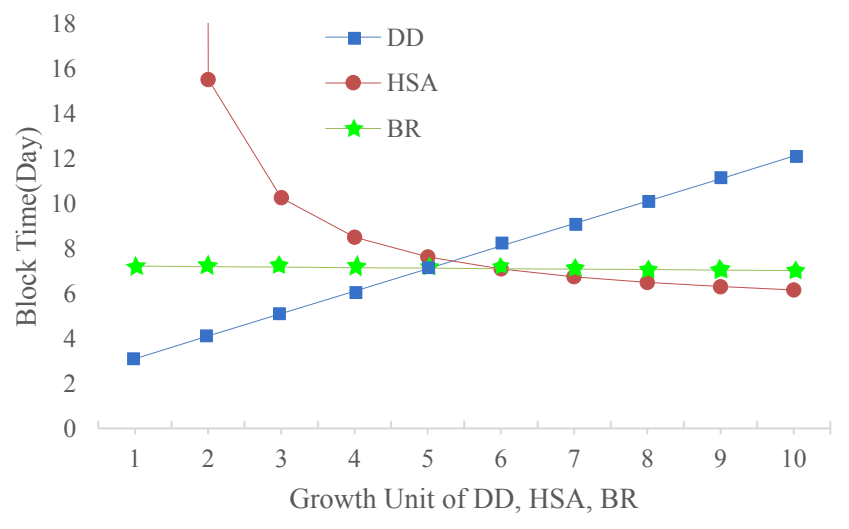

Figure 11: The factor influent block period

are set to be 1, 0.1 and 0.01, respectively. Figure 11 depicts the Block Time, whose $x$-axis represents the growth unit of DD, HSA and BR, and the y-axis denotes the blocking time. From this figure, we can see that DD has a linear positive correlation with blocking time. For the HSA, the lower the value, the greater the impact on blocking time. The higher DD and HSA, the blocking time is less affected by the BR.

535 The effect of the HSA on the blocking time follows a power function with a parameter of -1 , which means the lower a sender's historical reputation is, the more drastic effect will be exerted on the blocking time. Because the low historical reputation indicates that the sender is more frequently producing network anomalies, it will always be the object of vigilance, and the punishment 540 should be heavier. If the history reputation is high (there is rarely a network anomaly in history), the influence of HSA on blocking time is lighter, while DD 
plays a decisive role in the traffic accountability. The larger the DD is, the more serious the damage will be caused by the unwanted traffic, and therefore more timely measures to stop the loss are needed. accountable.

\subsection{Privacy}

Mix is an anonymous approach proposed by Chaum in [15. The essence of 565 Onion routing 16 algorithm uses source routing to randomly select three onion routers to form a transmission path and encrypt data for many times to effectively hide sender and receiver. However, the receiver cannot account for malicious traffic. AHP asks for ISP's assistance and uses NAT to map IP address to hide IP and stick IP to achieve privacy in 32. APIP 21] separated the accountability role from the source address. APNA 22] used Management Service (MS) to issue an EphID, which is used to replace sender address. However, those studies do not provide destination host privacy preservation, and the third-party runs the risk of leaking sender's privacy. Based on AIP and 575 APIP, 33. proposed a new architecture for trusted anonymous use of services in distributed computing networks.

\section{Conclusion}

This paper has tackled the latest research on credibility and privacy preservation in network layer and proposed PCNP. PCNP has introduced CVA to hide 
communicator's identity and performed trusted communication under the condition that communicators are anonymous to CVA. The security, deployment and compatibility of the proposed PCNP has been analyzed. The performance of PCNP has been validated by extensive experiments. The results have demonstrated the feasibility of the proposed PCNP.

\section{Acknowledgment}

This work is partially supported by the National Science and Technology Major Project of the Ministry of Science and Technology of China (Grant No.: 2017ZX03001019) and the Science and Technology Service Network Initiative (STS) Project of Chinese Academy of Science (Grant No.: Y7X0071105).

590 [1] J. Lopez, R. Rios, F. Bao, G. Wang, Evolving privacy: From sensors to the internet of things, Future Generation Computer Systems 75 (2017) 46 - 57. doi:10.1016/j.future.2017.04.045

[2] Y. Yang, X. Zheng, W. Guo, X. Liu, V. Chang, Privacy-preserving fusion of iot and big data for e-health, Future Generation Computer Systems 86 (2018) 1437 - 1455. doi:https://doi.org/10.1016/j.future.2018.01. 003 .

[3] Y. Ma, Y. Wu, J. Ge, J. Li, An architecture for accountable anonymous access in the internet-of-things network, IEEE Access 6 (2018) 14451-14461. doi:10.1109/ACCESS.2018.2806483

[4] S. Li, S. Zhao, P. Yang, P. Andriotis, L. Xu, Q. Sun, Distributed consensus algorithm for events detection in cyber physical systems, IEEE IoT Journal.doi:10.1109/JIOT.2019.2906157.

[5] H. Liu, H. Ning, Y. Zhang, L. T. Yang, Aggregated-proofs based privacypreserving authentication for v2g networks in the smart grid, IEEE Transactions on Smart Grid 3 (4) (2012) 1722-1733.

[6] H. Liu, H. Ning, Y. Zhang, Q. Xiong, L. T. Yang, Role-dependent privacy preservation for secure v2g networks in the smart grid, IEEE Transactions on Information Forensics and Security 9 (2) (2014) 208-220.

[7] Q. Liu, A. Srinivasan, J. Hu, G. Wang, Preface: Security and privacy in big data clouds, Future Generation Computer Systems 72 (2017) 206 - 207. doi:10.1016/j.future.2017.03.033

[8] Q. Wang, B. Qin, J. Hu, F. Xiao, Preserving transaction privacy in bitcoin, Future Generation Computer Systemsdoi:10.1016/j.future.2017. 08.026

${ }_{615}^{6}$ [9] L. Zhang, Z. Yan, R. Kantola, Privacy-preserving trust management for unwanted traffic control, Future Generation Computer Systems 72 (2017) 305 - 318. doi:10.1016/j.future.2016.06.036. 
[10] Rohan, Cybersecurity market worth 231.94 billion usd by 2022 .

cyber-security.asp

[11] P. Fischer, P. Stocken, Imperfect information and credible communication, Jour. of Accounting Res. 39 (1) (2010) 119-134.

[12] M. Reiter, A. Rubin, Crowds: Anonymity for web transactions, ACM Trans. Inf. Syst. Secur. 1 (1) (1998) 66-92. doi:10.1145/290163.290168.

625 [13] J. Boyan, The anonymizer - protecting user privacy on the web (1997).

[14] N. Ahmad, H. Cruickshank, Y. Cao, F. Khan, M. Asif, A.Ahmad, G. Jeon, Privacy by architecture pseudonym framework for delay tolerant network,

1. Future Generation Computer Systemsdoi:https://doi.org/10.1016/j. future.2017.11.017,

630 [15] D. Chaum, Untraceable electronic mail, return addresses, and digital 10 pseudonyms, Commun. ACM 24 (2) (1981) 84-90. doi:10.1145/358549. 358563.

[16] M. Reed, P. Syverson, D. Goldschlag, Anonymous connections and onion routing, IEEE Jour. on Selected Areas in Commun. 16 (4) (1998) 482-494. doi:10.1109/49.668972.

[17] R. Oppliger, Privacy protection and anonymity services for the world wide web (www) 16 (4) (2000) 379 - 391. doi:https://doi.org/10.1016/ S0167-739X (99) 00062-X.

[18] C. Gao, N. Iwane, A social network model for big data privacy preserving and accountability assurance, Consumer Commun. and NETWORKING Conf. IEEE (2015) 9-12doi:10.1109/CCNC.2015.7157940.

[19] J. Caverlee, L. Liu, Countering web spam with credibility-based link analysis, in: Proceedings of the Twenty-sixth Annual ACM Symposium on Principles of Distributed Computing, PODC '07, 2007, pp. 157-166. doi:10.1145/1281100.1281124.

[20] M. Afanasyev, T. Kohno, J. Ma, N. Murphy, S. Savage, A. Snoeren, G. Voelker, Privacy-preserving network forensics, Commun. ACM 54 (5) (2011) 78-87. doi:10.1145/1941487.1941508.

[21] D. Naylor, M. Mukerjee, P. Steenkiste, Balancing accountability and privacy in the network, SIGCOMM Comput. Commun. Rev. 44 (4) (2014) 75-86. doi:10.1145/2740070.2626306.

[22] T. Lee, C. Pappas, D. Barrera, P. Szalachowski, A. Perrig, Source accountability with domain-brokered privacy, Proc. of the 12th Inter. on Conf. on Emerging Networking EXperiments and Tech. 44 (4) (2016) 345-358. doi:10.1145/2999572.2999581. 
[23] S. Li, T. Tryfonas, H. Li, The internet of things: a security point of view, Internet Research 26 (2) (2016) 337-359.

[24] S. Li, S. Zhao, Y. Yuan, Q. Sun, K. Zhang, Dynamic security risk evaluation via hybrid bayesian risk graph in cyber-physical social systems, IEEE Transactions on Computational Social Systems 5 (4) (2018) 1133-1141.

[25] S. Bechtold, A. Perrig, Accountability in future internet architectures, Commun. ACM 57 (9) (2014) 21-23. doi:10.1145/2644146.

[26] Y. Zhang, L. T. Yang, J. Chen, Security in wireless sensor networks, RFID and sensor networks: architectures, protocols, security, and integrations (2009) 415-460 doi:10.1201/9781420077780.

[27] D. Raychaudhuri, K. Nagaraja, A. Venkataramani, Mobilityfirst: a robust and trustworthy mobility-centric architecture for the future internet, ACM (2012) 2-13.

[28] R. Moskowitz, P. Nikander, Host identity protocol (hip) architecture, RFC 4423 (2006) 1-24. doi:10.17487/rfc4423.

[29] S. Kent, C. Lynn, K. Seo, Secure border gateway protocol (s-bgp), IEEE Journal on Selected Areas in Commun. 18 (4) (2000) 582-592. doi:10. 1109/49.839934.

[30] D. Andersen, H. Balakrishnan, H. Feamster, T. Koponen, D. Moon, S. Shenker, Accountable internet protocol (aip), SIGCOMM Comput. Commun. Rev. 38 (4) (2008) 339-350. doi:10.1145/1402946.1402997.

[31] L. Zhang, Z. Yan, R. Kantola, Privacy-preserving trust management for unwanted traffic control, Future Generation Computer Systems, Elsevier 72 (2016) 305-318. doi:10.1016/j.future.2016.06.036.

[32] B. Raghavan, T. Kohno, A. Snoeren, D. Wetherall, Enlisting isps to improve online privacy: Ip address mixing by default, Privacy Enhancing Tech., Springer (2009) 143-163doi:10.1007/978-3-642-03168-7_9.

[33] Y. Ma, Y. Wu, J. Ge, J. Li, A new architecture for anonymous use of services in distributed computing networks, 2017 IEEE International Symposium on Parallel and Distributed Processing with Applications and 2017 IEEE International Conference on Ubiquitous Computing and Communications (ISPA/IUCC) (2017) 368-374doi:10.1109/ISPA/IUCC.2017.00059 